\title{
Optimization of Saddle Coils for Magnetic Resonance Imaging
}

\author{
Carlos Ernesto Garrido Salmon, Edson Luiz Géa Vidoto, Mateus José Martins, and Alberto Tannús \\ Instituto de Física de São Carlos, Universidade de São Paulo, CEP 13560-970, São Carlos, SP, Brazil
}

Received on 15 September, 2005; accepted on 28 November, 2005

\begin{abstract}
In Nuclear Magnetic Resonance (NMR) experiments, besides the apparatus designed to acquire the NMR signal, it is necessary to generate a radio frequency electromagnetic field using a device capable to transduce electromagnetic power into a transverse magnetic field. We must generate this transverse homogeneous magnetic field inside the region of interest with minimum power consumption. Many configurations have been proposed for this task, from coils to resonators. For low field intensity $(<0.5 \mathrm{~T})$ and small sample dimensions $(<30 \mathrm{~cm})$, the saddle coil configuration has been widely used. In this work we present a simplified method for calculating the magnetic field distribution in these coils considering the current density profile. We propose an optimized saddle configuration as a function of the dimensions of the region of interest, taking into account the uniformity and the sensitivity. In order to evaluate the magnetic field uniformity three quantities have been analyzed: Non-uniformity, peak-to-peak homogeneity and relative uniformity. Some experimental results are presented to validate our calculation.
\end{abstract}

Keywords: Magnetic ressonance imaging; Saddle coils

\section{INTRODUCTION}

In Nuclear Magnetic Resonance (NMR) experiments, besides the apparatus designed to acquire the NMR signal, it is necessary to generate a radio frequency electromagnetic field using a device capable to transform electromagnetic power into a transverse magnetic field. We should generate this transverse homogeneous magnetic field inside the region of interest (ROI) with the minimum power consumption or, using the reciprocity principle, with the maximum signal to noise ratio. There are two major classes of transducers, designated by coils when it is possible to separate from the resonating structure, normally a tank circuit, the inductive (magnetic field generating) parts from the capacitive parts. In another class the transducers are designated by resonators, meaning that the whole magnetic generating structure has to be thought of as a resonator.

Many configurations have been proposed for that function, from coils to resonators. For low field intensity $(<0.5 \mathrm{~T},<21$ $\mathrm{MHz}$ for $\left.{ }^{1} \mathrm{H}\right)$ and small sample dimensions $(<30 \mathrm{~cm})$, the saddle coil configuration has been used widely. The principal reason is its simplicity in both the construction and tuning processes. A saddle coil is represented by at least four conductors (called rungs or rods) on the surface of a circular cylinder oriented in the $\mathrm{z}$ direction. In this work we use the configuration sketched in Fig. 1. High order coils (i.e.: more than four rods) will not be analyzed in this paper. This basic configuration has four geometric parameters: diameter (D), length (Lo), angular aperture $(\alpha)$ and width of the conductors $(d)$.

The task to design such coils is to optimize the electromagnetic problem with two determinant factors: uniformity and efficiency. We must know the electromagnetic field distribution to estimate both factors. Therefore some electromagnetic model is assumed for the calculation. When the wavelength of the driving RF is large compared with the maximum dimension of the coil the retardation effects are negligible. The problem becomes a quasi-static calculation with an upper limit on the frequency. Thus the Biot-Savart law can be utilized for

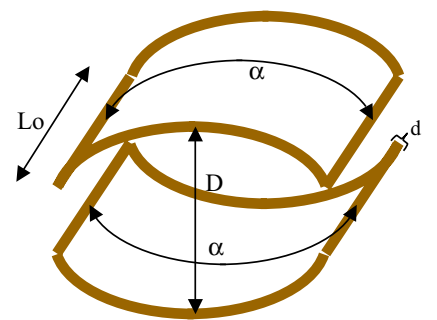

a)

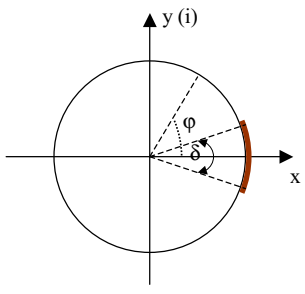

b)
FIG. 1: a) General sketch of a four finite width rungs saddle coil configuration. The letters indicate the geometric parameters: diameter, length, angular aperture and width of the conductors; b) Representation of a unique conductor on the surface coil in the complex plane $(\mathrm{z}=0)$; the polar angle $(\varphi)$ and the $\delta$ parameter are indicated in the figure.

the calculation of the magnetic field. On the other hand, when the conductors on the circumference of the coil are allowed to have widths of the same order of magnitude as the coil diameter, it is known that the conductors can no longer be approximated simply as single wires [1-3]. This is because the distribution of current within the finite-width conductors is not known, and must consequently be determined. The problem could be a one-dimensional and frequency independent problem regarding the skin depth much less than the thickness of the foil. Finally, if we restrict the frequency between upper and lower limits, then the current distribution depends only of the geometry of the conductor. Hence an optimization process considering only geometric parameters can be carried out.

Ginsberg and Melchner presented many years ago [4] an analytic solution $\left(\alpha=120^{\circ}, \mathrm{Lo} / \mathrm{D}=2\right)$ for the maximum uniformity in a simple saddle coil. However this solution is the optimal geometry with respect to the local uniformity of magnetic field only at the coil center. In spite of this fact many authors have used those values as optimal parameters [3, 5-7]. Hanssum determined another numerical solution improving 
the uniformity in a large volume, but he assumed a filament model for the current distribution [8]. Other authors have optimized for non-ideal conductors [1,2], only for a particular volume of interest. In some applications the sample almost fills the whole coil interior volume (e.g. in receptor coils) and in other cases the sample is small compared with the coil dimensions (e.g. in whole body transmission coils). Accordingly, it is useful to optimize the transducer, considering the relative sample dimensions.

In this paper we propose a simple analytic procedure to estimate the current density profile in finite width conductors in a saddle coil configuration. Using this profile we optimize the angular aperture calculating numerically the magnetic field distribution for different ROI dimensions. In the optimization process three quantities related to uniformity are evaluated. Each one of them will be defined in the next section.

\section{METHODOLOGY}

In this work, the four rungs are arranged around the circumference of a circular cylinder of diameter $\mathrm{D}=25.6 \mathrm{~cm}$ for head images (see Fig. 1-a). We use a method similar to that presented by other authors [1,2], using conformal mapping [9], to compute the current distribution within the finite width conductors. However, in order to obtain an analytic solution, a more simplified method was applied [10]. The electrostatic potential was calculated for an isolated rung considering the perturbation of other rungs as second order. This approach is valid when the rods are not very close to one another. Thus the transverse component of the electric field on the conductor surface is expressed (in the complex plane, Fig. 1-b) as:

$$
E_{t}(\varphi)=-\frac{\lambda \cdot \sqrt{1+c^{2}}}{\pi \cdot \varepsilon_{0} \cdot a} \frac{\cos (\varphi) \cdot \operatorname{sen}^{2}(\varphi)}{(1+\cos (\varphi)) \cdot \sqrt{\cos ^{2}(\varphi)-c^{2} \cdot \operatorname{sen}^{2}(\varphi)}} \cdot(\cos (\varphi)-i \cdot \operatorname{sen}(\varphi))
$$

where, $\varphi$ is the polar angle, $\lambda$ is the charge linear density and $\mathrm{c}$ is the width coefficient $\cot (\delta / 4)$. The rung width (d) was expressed in degrees, $\delta$ garameter, as the angle subtended on the circular perimeter. Null rung width (e.g. $\delta=0^{\circ}$ ) represents a wire with infinitesimal width, i.e., a filament model.

The resultant electric field at each point was calculated using the equation (1) and applying the superposition principle considering the four rungs in different angular positions. The final current density within each conductor has the same distribution as the normal component of the total electric field in each point. Thus, the current density is found according to the condition:

$$
J_{z}(\varphi)=\sqrt{\frac{\mu}{\varepsilon}} \cdot\left[\hat{n}_{1} \cdot \vec{E}_{t}(\varphi)-\hat{n}_{2} \cdot \vec{E}_{t}(\varphi+\alpha)-\hat{n}_{3} \cdot \vec{E}_{t}(\varphi-\pi)+\hat{n}_{4} \cdot \vec{E}_{t}(\varphi-\pi+\alpha)\right]
$$

Here, $\hat{n}_{i}$ denotes the normal to the conducting surface in each rod and the angular aperture $(\alpha)$ is the angular distance between the rod centers in the same saddle.

We applied the Biot-Savart law to calculate the magnetic field distribution inside of the ROI considering the current density distribution obtained in (2). The region-of-interest was defined by a 20 -cm-diameter circle located in the central transverse plane $(\mathrm{z}=0)$ of the coil. This ROI is similar to a human head. In order to evaluate the uniformity of magnetic field three quantities were computed: Non-uniformity (NU), peak-to-peak homogeneity (Inh) and relative uniformity (RU). These parameters are the more commonly used by many designers $[1,3,8,11]$. Curiously no one used more than one quantity on the uniformity evaluation. We calculate all of them because each one has its different meaning.

Non-uniformity is the root mean square deviation relative to mean field value $(\mathrm{Bm})$ :

$$
N U=\sigma / B m \cdot 100
$$

The peak-to-peak homogeneity is the difference between maximum and minimum values, expressed in percentage of the field at the coil center or center field $(\mathrm{Bc})$ :

$$
\operatorname{Inh}=(\text { Bmax }- \text { Bmin }) / B c \cdot 100
$$

The relative uniformity (RU) represents the percentage areas within $\pm 5 \%$ field deviation relative to $\mathrm{Bc}$ :

$$
R U=(\text { Points with } \Delta>5 / \text { Total points }) \cdot 100
$$

where $\Delta$ : 
$\Delta=|\mathrm{B}-\mathrm{Bc}| / \mathrm{Bc} \cdot 100(5)$

Two saddle coils with different angular aperture were constructed and compared (optimized and non-optimized, details in the next section). Both coils were used as receivers to acquire Magnetic Resonance images. The experimental magnetic field distribution was obtained from the images (SE, $\mathrm{TR} / \mathrm{TE}=2000 / 32 \mathrm{~ms}, \mathrm{FOV}=256 \mathrm{~mm}$ ) of a homogeneous cylindrical phantom $\left(2 \mathrm{mM} \mathrm{CuSO}_{4}\right)$. The visualization of the RF maps was improved using a median filter $8 \times 8$ combined with a noise threshold [12]. The measurements were carried out in a 0.5 T homemade MRI machine (ToRM 05) using a homogeneous whole-body RF coil for transmission.

\section{RESULTS AND DISCUSSION}

The angular aperture $(\alpha)$ and rung width $(\delta)$ were varied in a saddle configuration with a practical Lo/D ratio close to 1 . The magnetic field distribution in the ROI was determined using the computation procedure commented in the last section. The results are presented in the figures 2-5.

Different optimum aperture values for each uniformity quantity $\left(130^{\circ}\right.$ for NU, $125^{\circ}$ for RU and $135^{\circ}$ for Inh), can be noted from Figs 2-4. It suggests that each one doesn't represent the same result and so it have different meanings. The peak-to-peak homogeneity has more information about the more distant points in the ROI because it uses the maximum and minimum values (Equation 3). The relative uniformity is a criterion to evaluate the central area of the ROI because it is referred to the central field (Equations 4 and 5). The nonuniformity is a more global parameter. It contains information about the entire ROI (Equation 2), thus the standard deviation turns out to be a convenient procedure for field homogeneity evaluation as in magneto-static problems [13]. Accordingly, for coil design the meaning of each RF uniformity quantity must be taken in account, especially in large ROI.

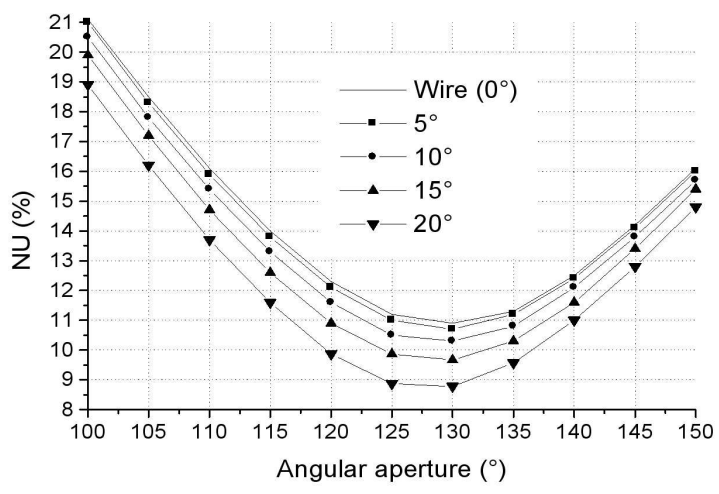

FIG. 2: Plot of the non-uniformity (NU) versus aperture angular for different rung width of the saddle coil described in the text.

For the three quantities, a larger conductor represents more uniformity. These findings are in agreement with a previous result [1]. The greatest improvement is $20 \%$ from wire to foil

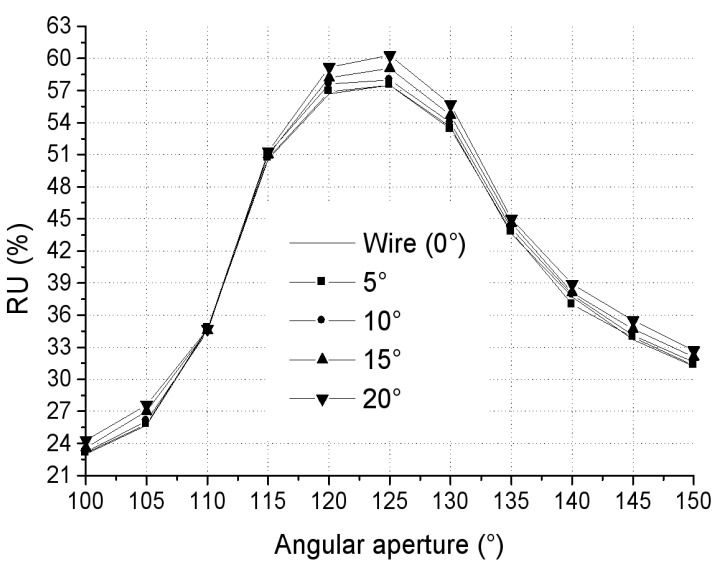

FIG. 3: Plot of the relative uniformity (RU) versus aperture angular for different rung width of the saddle coil described in the text.

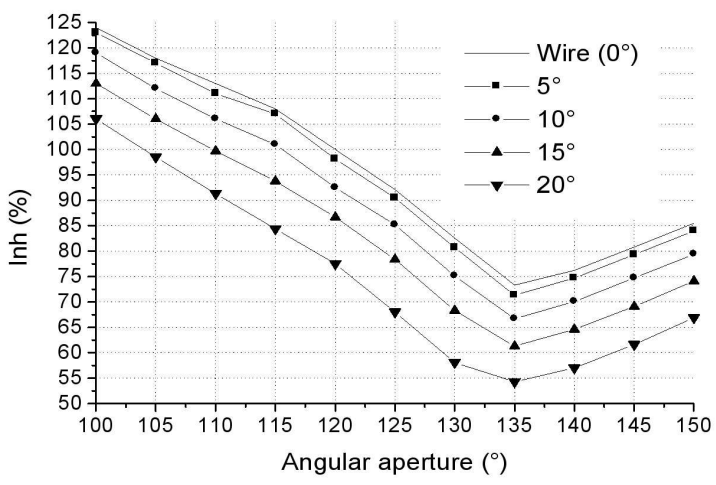

FIG. 4: Plot of the peak-to-peak homogeneity (Inh) versus aperture angular for different rung width of the saddle coil described in the text.

in the peak-to-peak homogeneity (Fig. 4). Also for all these quantities, the optimum angular aperture is independent of the rung width. Fig. 5 shows independence of the foil dimensions and the coil's sensitivity. This parameter is the field generated by current unit at the coil center $(\mathrm{Bc})$.

Another important factor in the RF coil design is the dimension of the ROI [8]. It can be noticed that all optimum apertures are different from the analytically predicted $120^{\circ}$. This fact is due to the ROI diameter (Di). In Fig. 6, the optimum aperture behavior is plotted as a function of ratio ROI/coil diameters using two uniformity quantities for finite and infinite length coils. In this case the rung width independence depicted on Figs. 2, 3 and 4 guarantees a general result. At infinite length and a small ROI, the optimum aperture tends to the analytical value. In small ROI both quantities have the same value, as we expected, but different values are obtained in larger ROI. In this last region the uniformity quantity effects are more sensitive. Additionally, the optimum values are larger. The small length effect (i.e. close to $1^{\circ}$ ) is almost in- 


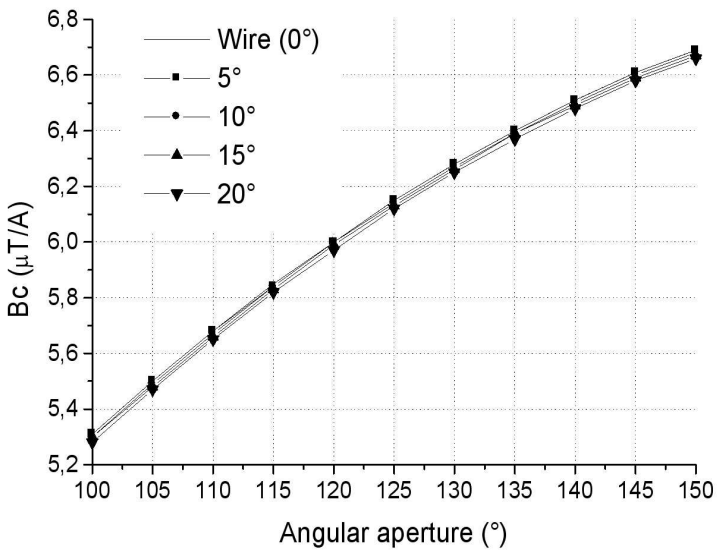

FIG. 5: Plot of the sensitivity (Bc, central field strength) versus aperture angular for different rung width of the saddle coil described in the text.

dependent of the ROI dimension and the uniformity quantity. Long coils must be constructed with larger aperture angles.

It is very difficult to define an optimum design due to the competition between the different factors (see figure 2 and 5). The uniformity and the sensitivity must be taken in account, but their relative weight is unknown. Accordingly, we imposed a trade-off. We defined a figure of merit considering the sensitivity six times more important than the non-uniformity. This function can be used in order to compare different designs. Fig. 7 shows the behavior of the figure of merit here defined, for a ROI with $60 \%$ of total circular area of the coil $(\mathrm{Di} \approx 0.77 \cdot \mathrm{D})$. So the optimum angular aperture for our case is around $135^{\circ}$.

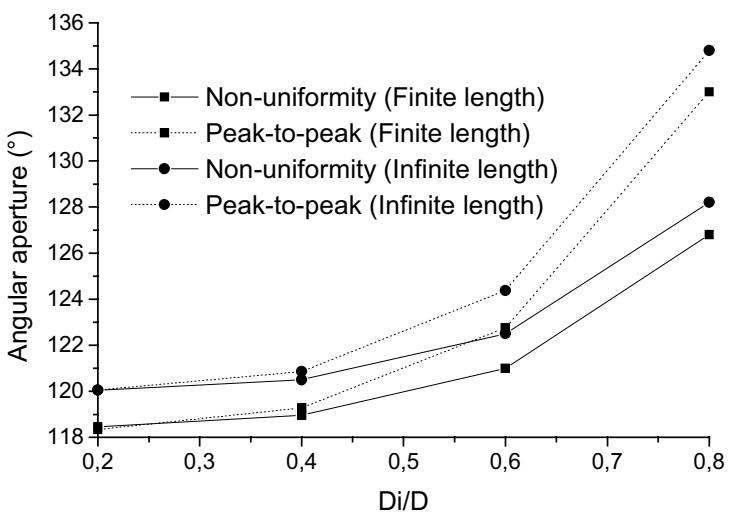

FIG. 6: Graph of the optimum angular aperture versus dimension of the ROI using two parameters for finite and infinite rods.

In order to test our calculus, two coils were constructed with similar length $(\approx 27 \mathrm{~cm})$, diameter $(\approx 26 \mathrm{~cm})$ and rung width $\left(\approx 15^{\circ}\right)$, but different angular aperture. The non-optimized coil had $108^{\circ}$ and the optimized coil had $135^{\circ}$. The figures 8 and 9 illustrate the theoretical and experimental maps of the magnetic field distribution. Besides the visual similarity be-

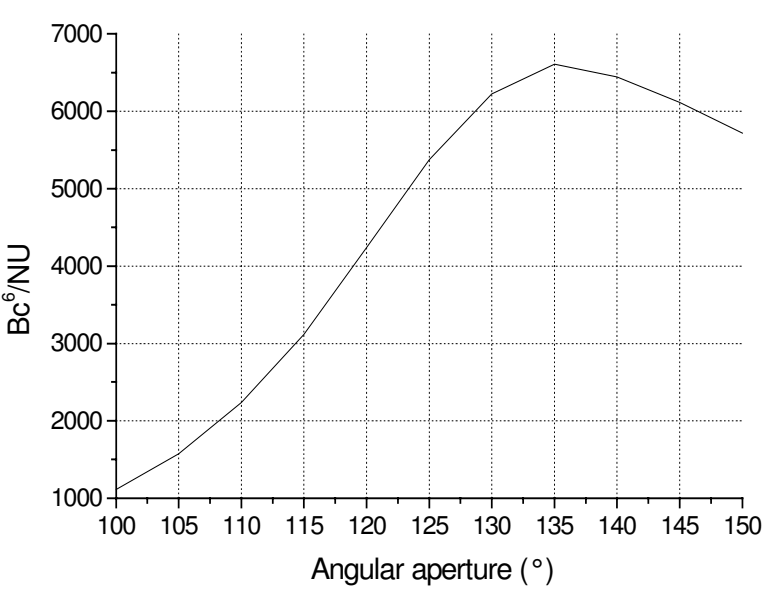

FIG. 7: Figure of merit as a function of angular aperture for $\mathrm{Di} \approx 0.77 \cdot \mathrm{D}$.

tween theoretical and experimental maps, a quantitative correspondence was obtained (Table I). Some differences could be attributed to the phantom effect because we do not consider the load.
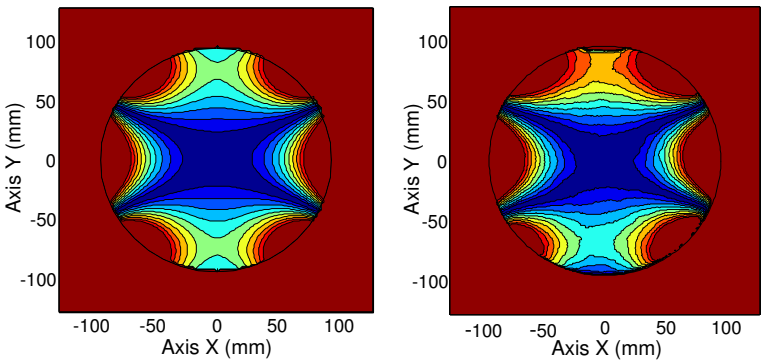

FIG. 8: Magnetic field distribution maps of the non-optimized coil (description in the text): a) Theoretic b) Experimental. Each contour represents $2 \%$ non-uniformity. The black circle delimited the ROI. The red region inside the ROI indicated non-uniformity larger than $20 \%$.

\section{CONCLUSIONS}

Different quantities can be defined to evaluate of RF field uniformity. The meaning of each quantity must be taken into account in the RF coil design, especially in large ROI. The non-uniformity using the root mean square deviation is the more global criterion for field uniformity evaluation.

The more sensible parameter in the saddle coil optimization is the angular aperture. In these coils the optimum angular aperture is independent of rung width; however, a larger conductor obtains slightly more uniformity, in agreement with a previous result [1]. The coil's length has a slight effect on the RF uniformity. Another important factor in the optimization process is the ROI size.

It is very difficult to define an optimum RF coil design. We 
TABLE I: Table I: Comparative results between theoretical and experimental magnetic field maps for optimized and non-optimized coils (Figs. 8 and 9).

\begin{tabular}{|c|c|c|c|c|}
\hline \multirow{2}{*}{ Quantity } & \multicolumn{2}{|c|}{\begin{tabular}{|l} 
Non-optimized $\left(108^{\circ}\right)$ \\
\end{tabular}} & \multicolumn{2}{|c|}{ Optimized $\left(135^{\circ}\right)$} \\
\hline & Theoretic & Experimental & Theoretic & Experimental \\
\hline $\mathrm{NU}(\%)$ & 15 & 14 & 10 & 9 \\
\hline RU (\%) & 34 & 33 & 41 & 46 \\
\hline $\operatorname{Inh}(\%)$ & 85 & 86 & 51 & 57 \\
\hline
\end{tabular}
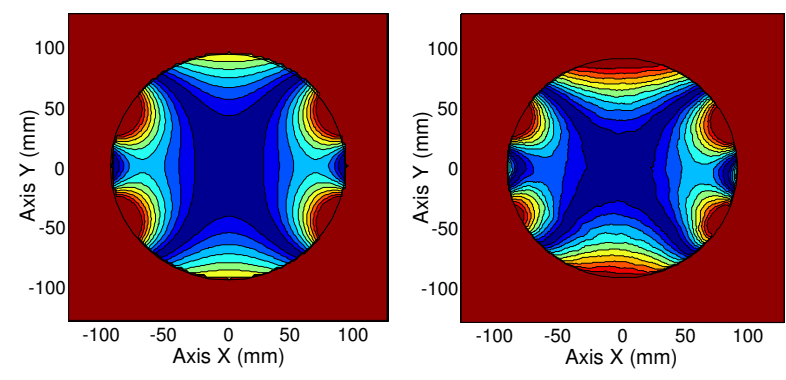

FIG. 9: Magnetic field distribution maps of the optimized coil (description in the text): a) Theoretic b) Experimental. Each contour represents $2 \%$ non-uniformity. The black circle delimited the ROI. The red region inside the ROI indicated non-uniformity larger than $20 \%$. propose a figure of merit considering the sensitivity six times more important than the non-uniformity. In this case for a ratio $\mathrm{ROI} /$ coil diameters close to 0.8 the optimum aperture is $135^{\circ}$.

We have demonstrated a simple model to determine the current density inside finite-width conductors in a four-rods saddle coil. The calculus model results were corroborated with experimental data.

\section{Acknowledgments}

The author CEGS is grateful to FAPESP Brazilian agency for partial financial support. We acknowledge José C. Gazziro and Odir A. Canevarollo for their involvement in constructing the RF coils.
[1] J. W. Carlson, Magn. Reson. Med. 3, 778 (1986).

[2] L. K. Forbes, S. Crozier, and D. M. Doddrell, Meas. Sci. Technol. 6, 284 (1995).

[3] S. Li, Q. X. Yang, and M. B. Smith, Magn. Reson. Imag. 12, 1079 (1994).

[4] D. M. Ginsberg and M. J. Melchner, Rev. Sci. Instr. 11, 122 (1970).

[5] R. Pérez de Alejo et al., Magn. Reson. Med. 51, 869 (2004).

[6] M. A. Macngauhtan et al., Anal Bional. Chem. 378, 1520 (2004).

[7] J. T. Heverhagen et al., MAGMA 17, 80 (2004).

[8] H. Hanssum, Appl. Phys. 19, 493 (1986).

[9] E. Durand, Électrostatique, Tome II Problèmes Généraux
Conducteurs. Paris, Mason et $\mathrm{C}^{i e}$, 1966. Cap.4, p.234-347: Grandeurs complexes et transformations conformes.

[10] C. E. Garrido, 2005. Development and clinical applications of a high performance radio-frequency/gradient coil integrated system for Magnetic Resonance Imaging in 0.5 Tesla. Appendix B. PhD. Thesis. Physical Institute of São Carlos. University of São Paulo.

[11] Ch. M. Collins et al., J. of Magn. Reson. 125, 233 (1997).

[12] K. P. Pruessman et al., Magn. Reson. Med. 42, 952 (1999).

[13] J. Teles, C. E. Garrido, and A. Tannús, J. Phys. D: Appl. Phys. 37, 1877 (2004). 